\title{
Powder bed laser sintering of copper-doped hydroxyapatite: Numerical and experimental parametric analysis.
}

François Rouzé l'Alzit ${ }^{1}$, Tiphaine Bazin ${ }^{1,2}$, Thierry Cardinal ${ }^{1}$, U-Chan Chung ${ }^{1}$, Sylvain Catros ${ }^{3}$, Caroline Bertrand ${ }^{1}$, Manuel Gaudon ${ }^{1 *}$, Gérard Vignoles ${ }^{4}$

${ }^{1}$ Université De Bordeaux, CNRS, Institut de Chimie de la Matière Condensée de Bordeaux (ICMCB), UMR 5026, Pessac, France.

${ }^{2}$ Université de Limoges, CNRS, Institut de Recherche sur les Céramiques, UMR 7315, Limoges, France

${ }^{2}$ Université De Bordeaux, INSERM, Bio-ingénierie Tissulaire (BioTis), UMR 1026, Bordeaux, France.

${ }^{4}$ Université de Bordeaux, Laboratoire des Composites Thermo Structuraux (LCTS), UMR 5801, Pessac, France.

*corresponding author: manuel.gaudon@icmcb.cnrs.fr / 0033540006685

\begin{abstract}
Additive manufacturing (AM), especially selective laser sintering (SLS), has been an increasingly popular field since the 1980s. Direct SLS is particularly challenging with ceramic materials due to their low thermal conductivity, high melting point, and brittle mechanical behaviour. In addition, they require longer processing times for a reliable sintering degree. In this work, the impact of some crucial parameters in SLS applied to a copper-doped hydroxyapatite bioceramic was studied. Incorporating copper ions into the hydroxyapatite matrix by thermal treatment can stimulate blood vessel formation, simultaneously improving the material's mechanical integrity and antibacterial properties. Moreover, it facilitates laser power absorption by the material at the laser wavelength. Thin films were prepared with
\end{abstract}


controlled thicknesses using dip-coating on glass substrates, and they were irradiated by an ytterbium laser (1.070 $\mu \mathrm{m}$ wavelength). Experimental laser irradiation was systematically compared to numerical models to predict the maximum temperature produced on the film surface by laser irradiation. The evaluated parameters are the absorptivity from the copper concentration, the film thickness, and the laser source (scanning velocity and power). Combining low laser scanning speeds with low film thickness and optimized absorption tuned by the copper concentration has made possible the development of well-sintered ceramics by the SLS technique.

\section{Keywords}

Selective laser sintering, Hydroxyapatite, Experimental analysis, Numerical model, Copper

\section{Introduction}

Calcium phosphate $(\mathrm{CaP})$ bioceramics are widely used in the bone regeneration field (orthopaedics and dentistry) due to their excellent biocompatibility, osteo-integration and osteo-conduction [1,2]. The most common $\mathrm{CaP}$ bioceramics include hydroxyapatite (HA), $\beta$ tricalcium phosphate $(\beta-\mathrm{TCP})$, and biphasic calcium phosphate $(\mathrm{BCP}$, constituted by an intimate mixture of HA and $\beta$-TCP). Hydroxyapatite is the primary inorganic constituent of bone $(\approx 60 \mathrm{wt} \%)$ and the main constituent of tooth enamel (90 wt $\%)$. HA, with the chemical composition $\mathrm{Ca}_{10}\left(\mathrm{PO}_{4}\right)_{6}(\mathrm{OH})_{2}$, possesses a hexagonal crystallographic structure that allows the incorporation of various doping ions in substitution of $\mathrm{Ca}^{2+}, \mathrm{P}^{5+}\left(\mathrm{PO}_{4}^{3-}\right)$ or $\mathrm{OH}^{-}$sites. This material's substitution capacity can be exploited to modify the biological response [3-5]. Elements naturally present in the body, such as silica, copper or iron, can be introduced into the HA structure. Copper is of particular interest because it stimulates blood vessel infiltration into the implants, accelerating bone regeneration [6]. In addition, the incorporation of copper ions in the HA matrix could eventually improve the mechanical integrity and antibacterial capacity of coatings for biomedical applications [7,8]. Along with $\mathrm{CaP}$ bioceramic synthesis development, the additive manufacturing (AM) of bioceramic parts has been studied since the 1980s [9-11]. Additive manufacturing (or 3D printing) is a process in which an architecture model, initially generated using a three-dimensional computer-aided design (3D CAD) system fabricated directly. In AM, the parts are made by adding material layer by layer, with each layer 
being a thin cross-section of the part derived from the original CAD dataset [12]. Among the AM techniques, selective laser sintering (SLS) uses a high-power laser beam that selectively irradiates a powder bed to produce localized thermal sintering. While the material partially absorbs at the laser source wavelength, irradiation induces locally elevated temperatures, allowing sintering to occur, i.e., powder particle sintering without melting (the matter typically remains in the solid-state). The sintering process can be divided into three stages. During the first stage, the formation of sintering necks leads to mechanical cohesion of the powder pack. In the second stage, grain growth and a reduction in the volume of the porosity network volume are observed. In the third stage, almost all spherical and isolated pores are eliminated $[13,14]$. Traditionally, SLS has been researched on a wide range of materials, but polymers (PVC and PEEK) or metals are preferred because they have relatively low melting points, are not fragile, and metals exhibit a high thermal conductivity. For bioceramics, sintering by the SLS process is particularly challenging due to the low thermal conductivity of ceramics, high melting point, brittle mechanical behaviour and longer time required for precise sintering [10,15]. Hussein et al. [16] observed that the irradiated zone tends to quickly shrink during cooling after the laser beam has left the area. Consequently, the high tensile stresses generated may also lead to crack formation. Crack formation in the areas submitted to compressive and tensile stresses occurs when the material's compressive yield stress is exceeded. Dense HA ceramics (up to $98 \%$ of their theoretical density) can be obtained by standard sintering at $1000-1200^{\circ} \mathrm{C}$ for a few hours. Processing at higher temperatures may lead to excessive grain growth and HA decomposition, which becomes unstable above $1250-1300^{\circ} \mathrm{C}$ [2]. Therefore, HA is mostly expanded using indirect laser sintering, i.e., ceramic materials are often combined with a low melting point material such as a polymer that serves as a binder [17-19]; thus, the intermediate 3D cohesive shape obtained from indirect SLS is post-treated in standard furnaces to achieve high density. The SLS process relies on many different parameters, roughly coming from two groups: the parameters closely related to the processed material and the used apparatus parameters. Yadroistsev et al. [20] suggested an index of process variables:

(a) Powder: composition, size distribution, shape, optical and heat transfer properties, and the thickness of the deposited layer.

(b) Laser: power, spot size, spatial beam distribution, scanning velocity, and application of protective gas atmosphere.

These main variables have an impact on the laser beam temperature. All the parameters must be clearly identified, and their impact must be evaluated to optimize selective laser sintering. However, in situ studies aiming to quantify the spatial temperature distribution reached from 
the matter/laser interaction are very complex to perform. Such experimental studies are also time-consuming because of the high number of key parameters to evaluate. Consequently, modelling constitutes an efficient alternative to assess the temperatures reached during the process and how they are impacted by the different materials or laser parameters [21,22]. It allows predicting the heat treatment regime with relatively high accuracy occurring during the experimental SLS process. For instance, Dong et al. [23] exhibited that their predictions were in good agreement with the experimental results. Several experiments were performed on heat conduction models to explore thermal behaviour during the SLS process for metal materials. Tolochko et al. [24] studied the heat transfer mechanism induced by laser irradiation on titanium powder by coupling an experimental procedure and a heat transfer simulation. Using this method, they explained that a nearly hemispherical front propagates from the laser spot. Three main powder parameters influence the sintering: (i) the contact thermal conductivity depending on the powder bed surface density, (ii) the radiative thermal conductivity, which can be adjusted by particle size, and (iii) the volumetric specific heat, which can be modified by the powder bed density and thickness. The last parameter is of the highest importance among these three parameters since the sintered or melted zone volumes decreased with the powder density reduction. Gusarov et al. [25] employed numerical methods to study a powder layer's absorbance on different metals $(\mathrm{Cu}, \mathrm{Fe}, \mathrm{Pb}, \mathrm{Ti})$. They confirmed that the absorbance of a powder bed is defined by a universal function of the absorptivity of the solid phase itself depending on the wavelength [26] and is independent of the powder particle morphology and the powder bed porosity. In contrast, Li et al. [27] analysed the thermal behaviour during selective laser melting (SLM) of aluminium powder and observed that the maximum temperature reached during the process is closely linked to film thickness and evolves with the number of layers. This is mainly due to the heat accumulation phenomenon and energy losses by conduction being higher than convection/radiation losses during the SLM process.

Most recently, some authors [28-32] have shown modelling results combined with experimental measurements on materials other than metals, such as polymers. The calculation results agreed well with the experimental measurements. Thus, numerical modelling is appropriate to simulate thermal diffusion inside the processed polymer layer during the SLS process.

To the best of our knowledge, no modelling study to simulate the thermal distribution for the SLS process applied to ceramics, especially bioceramics, has been reported thus far.

The aim of this work was to study the impact of each critical parameter in laser sintering of copper-doped HA films, whether they are linked to the powder material or the apparatus. 
Parameters linked to the Cu-doped HA (film absorption coefficient and film thickness) and parameters linked to the apparatus (especially laser power and laser writing speed) are both investigated. This paper focused on the parameter influence on the temperature distribution around the irradiated area and its evolution once irradiation is stopped. The numerical results obtained from an accurate model established using finite element analysis (FEA) software were compared to experimental observations (mainly SEM microscopy) on irradiated $\mathrm{Cu}$-doped HA films.

\section{Experimental}

\subsection{Powder preparation}

The ceramic powder used for the experiments (Cu-doped HA) was synthetized by solid-state process. Firstly, pure HA was prepared by co-precipitation. The protocol, details of which are reported elsewhere [33], consists of adding, at a controlled rate and a controlled $\mathrm{pH}$ thanks to ammonia $\mathrm{NH}_{4} \mathrm{OH}$, a phosphate solution (di-ammonium hydrogen-phosphate: $\left(\mathrm{NH}_{4}\right)_{2} \mathrm{HPO}_{4}$ ) to a calcium solution (obtained from dissolution of calcium nitrate: $\mathrm{Ca}\left(\mathrm{NO}_{3}\right)_{2}, 4 \mathrm{H}_{2} \mathrm{O}$ ). The quantities of the reagents were calculated to obtain a molar ratio $\mathrm{Ca} / \mathrm{P}=10 / 6$. The synthesis conditions set were a $\mathrm{pH}=8.5$ and a temperature maintained at $85^{\circ} \mathrm{C}$. The synthesis was performed under argon during a maturation period of 5 hours. The as-prepared precipitate was then centrifugated and washed with distilled water, then dried in an oven at $100^{\circ} \mathrm{C}$. The dried powder was calcined at $600^{\circ} \mathrm{C}$ for $1 \mathrm{~h}\left(10^{\circ} \mathrm{C} \cdot \mathrm{min}^{-1} \mathrm{ramp}\right)$ to remove reaction by-products such as nitrate groups. Next, a quantity of copper oxide (II) was mixed to the HA powder to obtain a copper concentration $x_{\mathrm{Cu}}=0.1,0.3,0.5$ or 0.7 in the formula $\mathrm{Ca}_{10} \mathrm{Cu}_{x}\left(\mathrm{PO}_{4}\right)_{6} \mathrm{O}_{2 x}(\mathrm{OH})_{(2-2 x)}$. The $\mathrm{HA}-\mathrm{CuO}$ powder mixture was crushed by attrition to reduce particle size and further promote solid-state reaction. Attrition was performed in a $100 \mathrm{~mL}$ Teflon bowl in the presence of approximately $50 \mathrm{~mL}$ of distilled water, $1 \mathrm{wt} \%$ Darvan $\mathrm{CN}$ dispersant and $75 \mathrm{~g}$ yttriumstabilized zirconia balls of $1 \mathrm{~mm}$ diameter. Attrition speed of $800 \mathrm{rpm}$ was applied for 3 hours. The suspension was dried in an oven at $100^{\circ} \mathrm{C}$. The resulting powder were pressed into pellets of $10 \mathrm{~mm}$ diameter by applying an uniaxial pressure of $100 \mathrm{MPa}$. Finally, the pellets were calcined (ramp $10^{\circ} \mathrm{C} \cdot \mathrm{min}^{-1}$ ) for 1 hour at a temperature of $1100^{\circ} \mathrm{C}$ to obtain a pure $\mathrm{Cu}$-doped HA phase.

\subsection{Thin films preparation}


The resulting pellets from powder synthesis were firstly re-crushed in a $100 \mathrm{~mL}$ Teflon bowl in the presence of approximately $50 \mathrm{~mL}$ of ethanol, $2 \mathrm{wt} \%$ Beycostat C213 dispersant and $75 \mathrm{~g}$ of yttrium-stabilized zirconia balls, with an attrition speed of $800 \mathrm{rpm}$ for 6 hours. After separation from the zirconia balls, the suspension contained $30 \mathrm{wt} \%$ of $\mathrm{Cu}$-doped HA powder and was then used for film deposition by dip-coating.

The dip-coating method was used to coat a silica glass substrate with a drawing speed of $400 \mathrm{~mm} \cdot \mathrm{min}^{-1}$. A film of a few microns thickness $(<5 \mu \mathrm{m})$ is usually obtained. To increase the total thickness of the film, 2 to 5 successive layers were deposited, with a drying step of the asprepared film at $100^{\circ} \mathrm{C}$ in between each coating step.

\subsection{Powder and film characterization}

Structural Properties. Powder or film X-ray diffraction (XRD) patterns were recorded on a PANalitycal X'pert PRO MPD diffractometer in Bragg-Brentano $\theta-\theta$ geometry equipped with a secondary monochromator and $\mathrm{X}^{\prime}$ Celerator multi-strip detector. Each measurement was made within an angular range of $2 \theta=8-80^{\circ}$ a $0.017^{\circ}$-step scan rate and counting time of $59 \mathrm{~s}$. The $\mathrm{Cu}-\mathrm{K} \alpha$ radiation was generated at $45 \mathrm{KV}$ and $40 \mathrm{~mA}(\lambda=0.15418 \mathrm{~nm})$.

Optical properties. The UV-visible-NIR powder absorption spectra were measured with a double-beam spectrophotometer (CARY 5000 UV-vis-NIR) over the 250-2500 nm spectral region.

Morphological characterization. The morphology of the samples was observed using a Leica DMI $3000 \mathrm{M}$ optical microscope equipped with an N PLAN EPI 10× objective. SEM investigations were performed using a JEOL JSM-67 in electron back-scattering mode.

\subsection{Selective laser sintering facilities}

The SLS machine used for the experimentation was a continuous ytterbium fiber laser with an emission wavelength of $1.070 \mu \mathrm{m}$, which has been especially designed according to the laboratory specifications by ES Laser (ES17-355). This equipment has a focal distance of $199 \mathrm{~mm}$, a spot size of $50 \mu \mathrm{m}$ and its maximum and minimum operating power is $400 \mathrm{~W}$ and $40 \mathrm{~W}$ respectively. Experiments were carried out in air, using thin films on glass substrates as samples on a fixed sample stage. The 2 modulating parameters used in the experiments are the power (expressed as a \% of maximum power) and the scanning speed (in $\mathrm{m} . \mathrm{s}^{-1}$ ). 


\subsection{Numerical simulation equations}

The modelling approach is focused on heat transfer from the laser to the film and from the film to its environment. It does not consider single particles but treats instead the powder film as a continuum with homogenized properties. Moreover, since the film thickness $d$ is much smaller than the other dimensions of the problem, it is chosen to restrict the model to a $2 \mathrm{D}$ depth-averaged model. The surface temperatures reached upon laser heating depend mainly on thermal conductivity, air/film interface convective and radiative losses, as depicted by the heat balance equation:

$\rho C p \frac{\partial T}{\partial t}=\operatorname{div}(k \nabla T)+Q_{\text {laser }}-H_{l}$

where $\rho$ is the material density $\left(k g . \mathrm{m}^{-3}\right), C p$ is the specific heat capacity $\left(\mathrm{J}^{\mathrm{kg}} \mathrm{g}^{-1} \cdot \mathrm{K}^{-1}\right), T$ is the temperature $(K), t$ is the interaction time $(s), k$ is the thermal conductivity of material $\left(W \cdot m^{-1} \cdot K^{-1}\right), Q_{\text {laser }}$ is the thermal volumetric power from laser irradiation $\left(W \cdot m^{-3}\right)$ and $H_{l}$ is the sum of the convective and radiative heat losses $\left(W \cdot m^{-3}\right)$.

The interaction of the laser beam with the material induces a Gaussian distribution of energy at the surface of the powder bed given by equation $\left(W \cdot m^{-2}\right)$ :

$Q_{0}(x, y)=a \frac{P}{\pi r^{2}} \exp \left(-\frac{x^{2}+y^{2}}{2 r^{2}}\right)$

where $a$ (ratio of the luminescence of the material and the luminescence of the black body for a same temperature) is the surface emittance (or absorptance) of the material, $P$ is the incident laser power $(W)$ and $r$ is the radius of the laser spot $(m)$.

The resolution domain is chosen to be large enough with respect to the spot size and track length, so that it is possible to assign simple adiabatic boundary conditions on its edges.

The power distribution in the material depends on the in-depth attenuation showing to follow the Beer-Lambert law [28]:

$Q(x, y, z)=Q_{0}(x, y) \exp (-\alpha z)$

where $\alpha$ is the extinction coefficient depending on the material, this can lead to two different situations. For small values of $\alpha\left(\ll d^{-1}\right)$, the shape of the heated volume in depth will almost be cylindrical. In the opposite situation, we can consider it as a semi-ellipsoid defined by its vertical axis: $\alpha^{-1}$ or a cylinder defined by its depth: $d$.

So, using an in-depth average, the mathematical expression for $Q_{\text {laser }}$ becomes Eq. (4) for a semi-ellipsoid or Eq. (5) for a cylinder. 
$Q_{\text {laser }}\left(x^{\prime}, y\right)=\frac{3 \alpha}{2} Q_{0}\left(x^{\prime}, y\right)$

$Q_{\text {laser }}\left(x^{\prime}, y\right)=\frac{Q_{0}\left(x^{\prime}, y\right)}{d}$

where $x^{\prime}=x-v t, v$ being the scanning speed. Convective and radiative heat losses are detailed as:

$H_{l}=\left[h\left(T-T_{\text {ext }}\right)+a \sigma\left(T^{4}-T_{\text {ext }}^{4}\right)\right] /\left(d\right.$ or $\left.\frac{2}{3 \alpha} r\right)$

where $h$ is the convective heat transfer coefficient between the powder bed surface and the air $\left(10 \mathrm{~W} \cdot \mathrm{m}^{-2} \cdot \mathrm{K}^{-1}\right)$ and/or glass $\left(1.2 \mathrm{~W} \cdot \mathrm{m}^{-2} \cdot \mathrm{K}^{-1}\right), T_{\text {ext }}$ the ambient temperature $(293 \mathrm{~K}), a$ the emissivity and $\sigma$ the Stefan-Boltzmann constant $\left(5 \cdot 67 \cdot 10^{-8} W^{-2} \cdot \mathrm{m}^{-4}\right)[20,29,33]$. However, in practice, the importance of the convective heat loss is demonstrated to be negligible, as shown in Appendix.

The numerical solution of the 2D transient heat equation (Eq. (1)) has been achieved using the FlexPDE Multiphysics Finite Element solver ${ }^{1}$.

\section{Results and discussions}

\subsection{Experiment design}

A standard set of numerical parameters was defined as irradiation is performed with a laser power of $60 \mathrm{~W}$ and a laser scan speed of $0.30 \mathrm{~m} . \mathrm{s}^{-1}$ on a Cu-doped $\mathrm{HA}$ film with $x_{\mathrm{Cu}}=0.1$ (i.e., with an $\alpha$ absorptivity coefficient equal to 0.08 (8\%)) deposited as 2 successive layers (with a thickness of $25 \mu \mathrm{m}$ ).

First, both the heat-loss coefficients linked to radiative loss of the $\mathrm{Cu}$-doped HA and the air/film interface convection heat transfer were assessed.

Then, the following four parameters of the as-prepared ceramics were selected due to their influence on the study: (i) the absorption coefficient of the material, (ii) the film thickness, (iii) the laser power, and (iv) the laser scanning velocity. Experiments were carried out modifying only one parameter at the time.

The film thickness parameter was altered from the number of layers ( 2 or 5 successive layers were used). For the absorption parameter, two various copper concentrations ( $x=0.1$ and $\mathrm{x}=0.5$ ) were used. The laser power and laser speed are inter-correlated since the line energy applied

\footnotetext{
${ }^{1}$ FlexPDE Lite v7.16, distributed by PDESolutions (www.pdesolutions.com)
} 
from the laser to the sample was directly proportional to the laser power/laser speed ratio. The aim was to assess whether the temperatures reached were dependent on the line energy by maintaining single linear energy (in $\mathrm{J}^{\mathrm{m}}{ }^{-1}$ ) while working with different power/velocity couples.

\subsection{Cu-doped HA properties}

Figure 1 shows the X-ray diffraction (XRD) patterns of $\mathrm{Ca}_{10} \mathrm{Cu}_{x}\left(\mathrm{PO}_{4}\right)_{6} \mathrm{O}_{2 x}(\mathrm{OH})_{(2-2 x)}$ with $x=$ 0.5 after annealing treatments at different temperatures. The "CuO-HA raw powder" (i.e., before any annealing treatment), constituted as a powder mixture of $\mathrm{CuO}$ and pure $\mathrm{HA}$ in stoichiometric proportion, exhibits diffraction peaks relative to both $\mathrm{CuO}$ and $\mathrm{HA}$ phases. Heat treatment of the raw mixture leads to a substitution of hydrogen atoms by copper, and the pure phase is formed at $1100^{\circ} \mathrm{C}$.
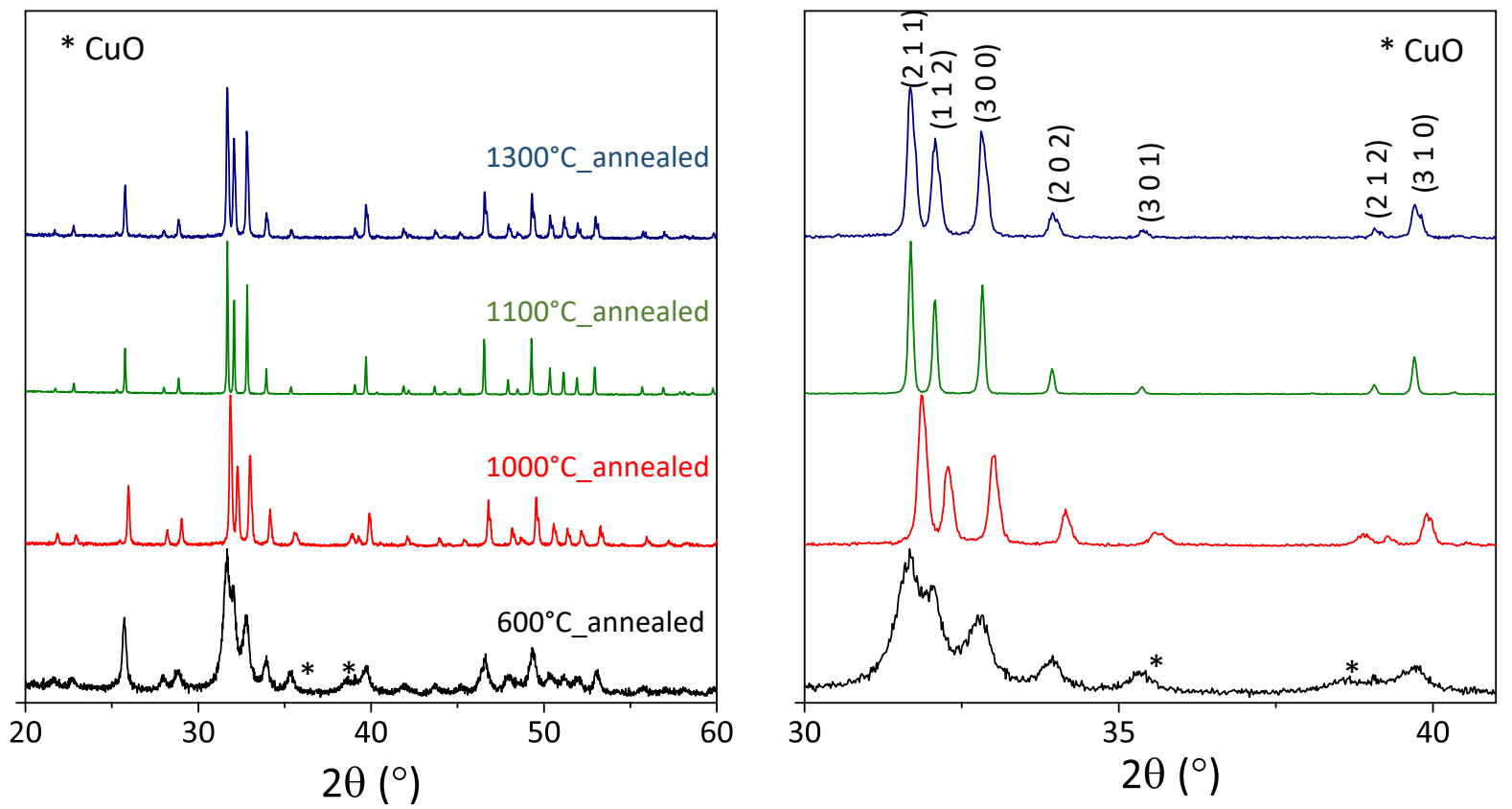

Figure 1. X-ray diffraction patterns of $\mathrm{CuO}-\mathrm{HA}, x=0.5$ after thermal treatment at different temperatures.

In Figure 2, the diffraction patterns obtained for $x=0.1,0.3,0.5$, and 0.7 are reported. For each composition, only HA structure diffraction peaks were detected, showing that copper cations were located inside the structural network, leading to a pure HA crystalline network.

Moreover, heat treatment leads to an evolution of the material's optical properties (Figure 3a, for illustration at the chemical composition $x=0.5$ ). The colour of the material changed with increasing heat treatment temperature due to the incorporation of copper inside the apatite 
network and copper oxide's concomitant disappearance. Thus, the colour evolved from grey to purple (Figure 3b).

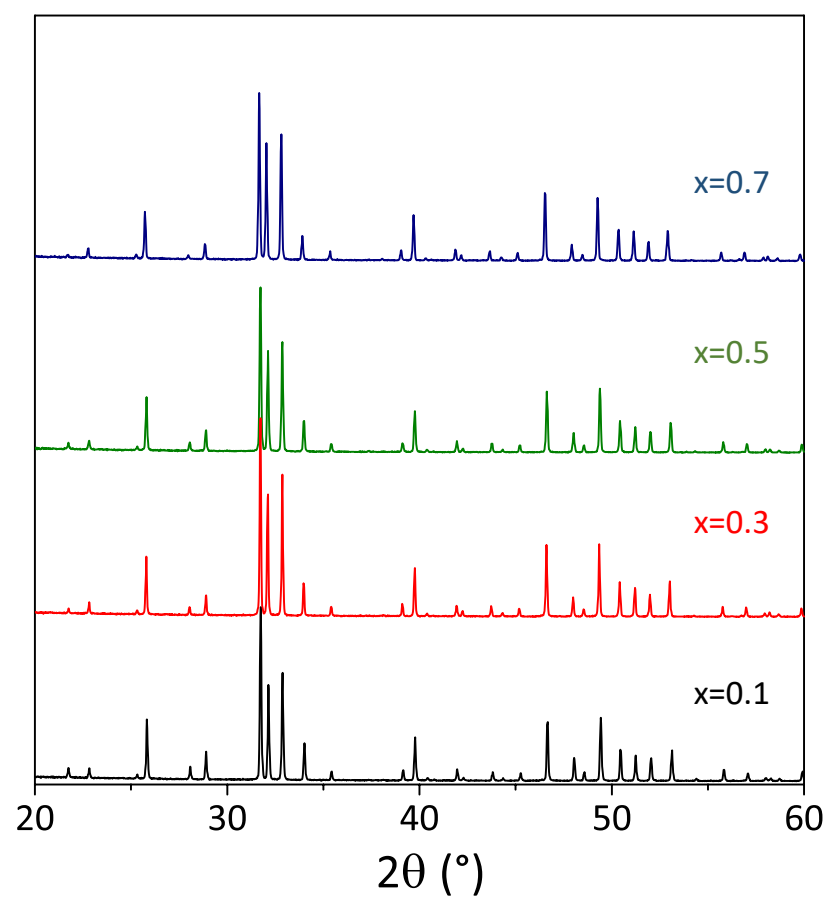

Figure 2. X-ray diffraction patterns of Cu-doped HA with different copper contents after thermal treatment at $1100^{\circ} \mathrm{C}$.

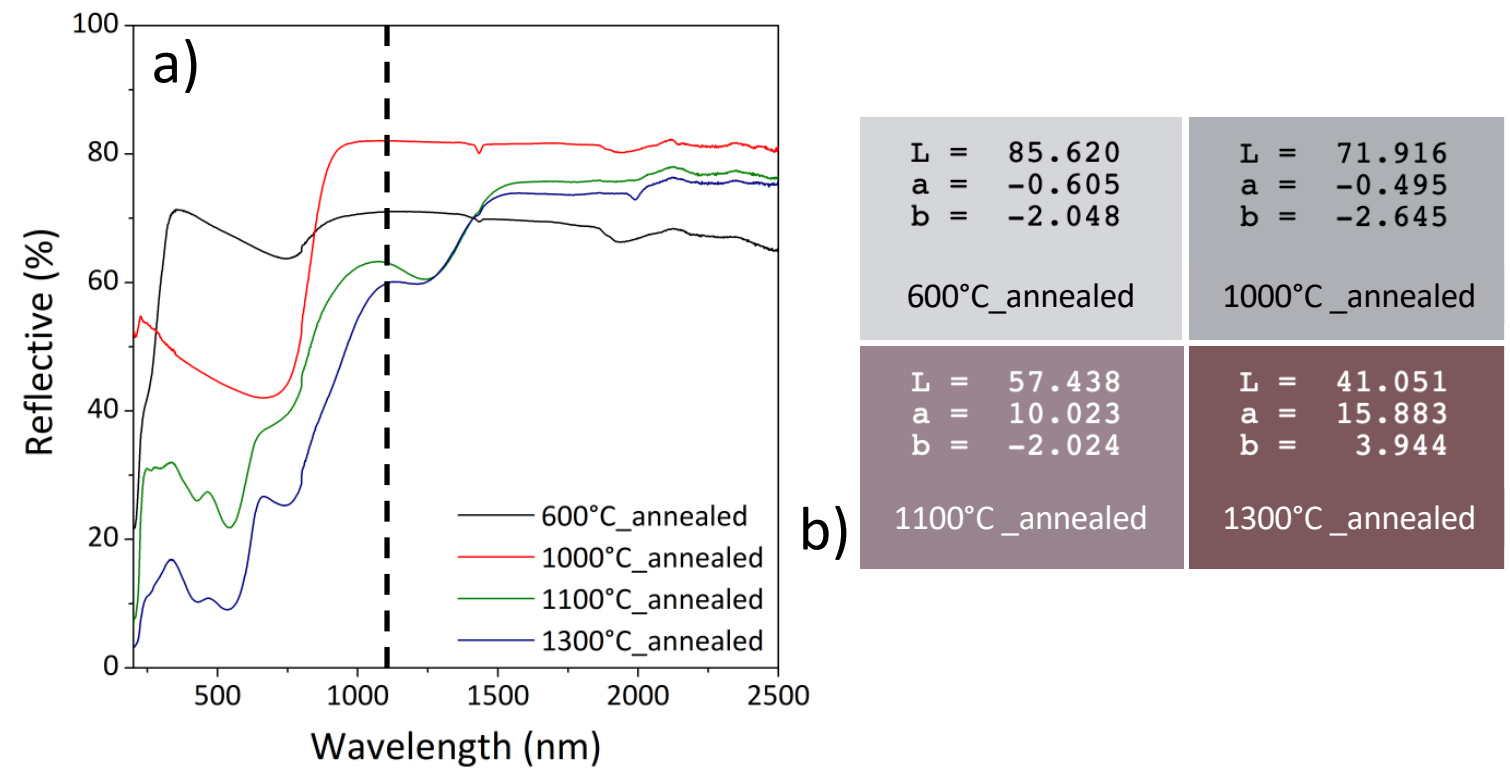

Figure 3. Diffuse reflectance spectra of Cu-doped HA with (a) $x=0.5$ obtained after thermal treatment at varying temperatures, and (b) corresponding $L^{*} a * b$ colorimetric parameters. 


\subsection{Impact of absorptance}

The absorptance at the sample laser wavelength was the first studied parameter. The results obtained by diffuse reflectance from different material samples with different copper concentrations revealed an increase in the copper concentration inserted in the HA matrix led to a linear increase in the absorption percentage at the laser wavelength (Figure 4).
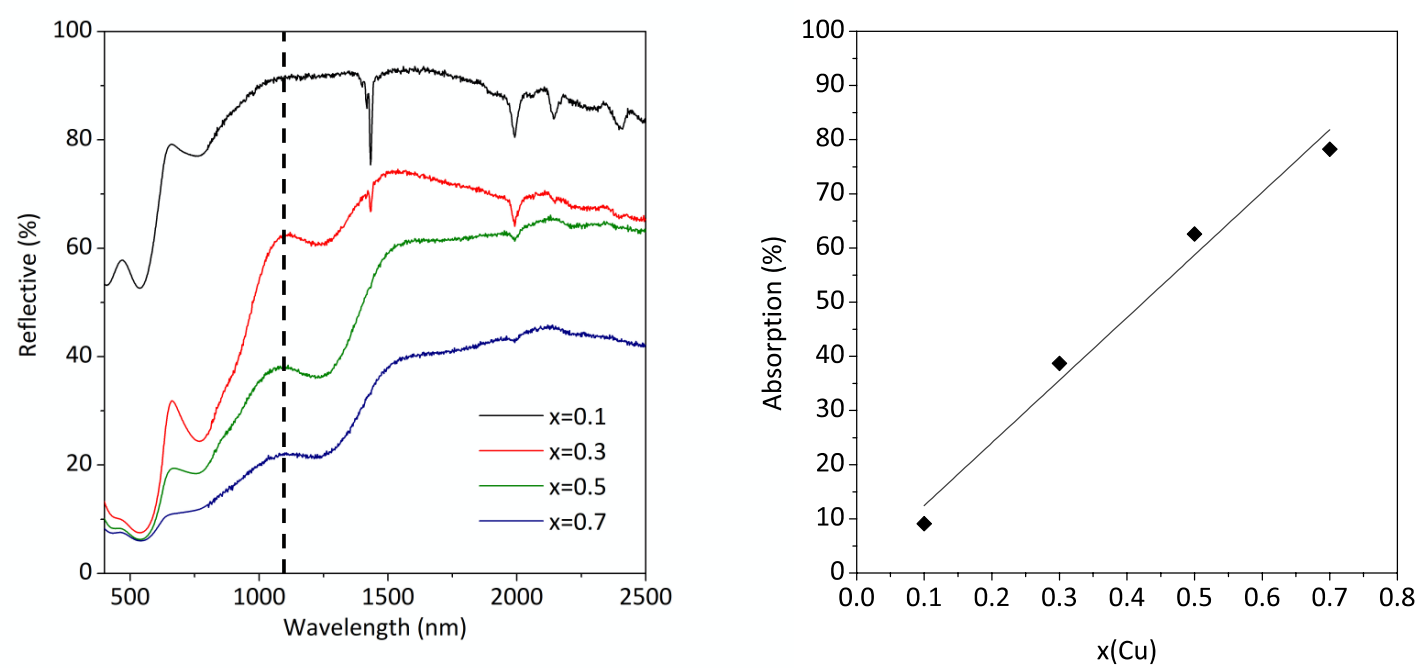

Figure 4. Evolution of absorption (\%) at the laser wavelength versus the concentration $x$ of copper in HA.

Two Cu-doped HA samples with different copper concentrations were prepared for comparison as thin films with a similar thickness (obtained from 2 successive dip-coating depositions). The laser-material interaction created diverse phenomena as a function of the HA film absorptance, as presented below. The subsequent studies were performed using the same laser scanning speed and laser power (standard laser parameters).

On the one hand, for the low copper concentration sample $(x=0.1)$, the laser/material interaction induced no sample colour change (as observed with an optical microscope: top images in Figure 5). SEM micrographs (bottom images in Figure 5) prove a powder bed was exhibiting particles without forming sintering necks between them. This implies a weak sintering effect due to a small thermal increase. Conversely, the sample with the higher absorptance (with the most significant copper amount: $x=0.5$ ) showed a change in colour observable with an optical microscope; spherical defects associated with bubble formation indicate significant thermal elevation leading to partial melting of the powder bed. SEM investigations confirmed that the melting point of HA was surpassed. Hence, the laser irradiation of these two $\mathrm{Cu}$-doped $\mathrm{HA}$ 
samples $(x=0.1$ and 0.5$)$ indicated the drastic impact of the absorptance on the achieved temperatures.
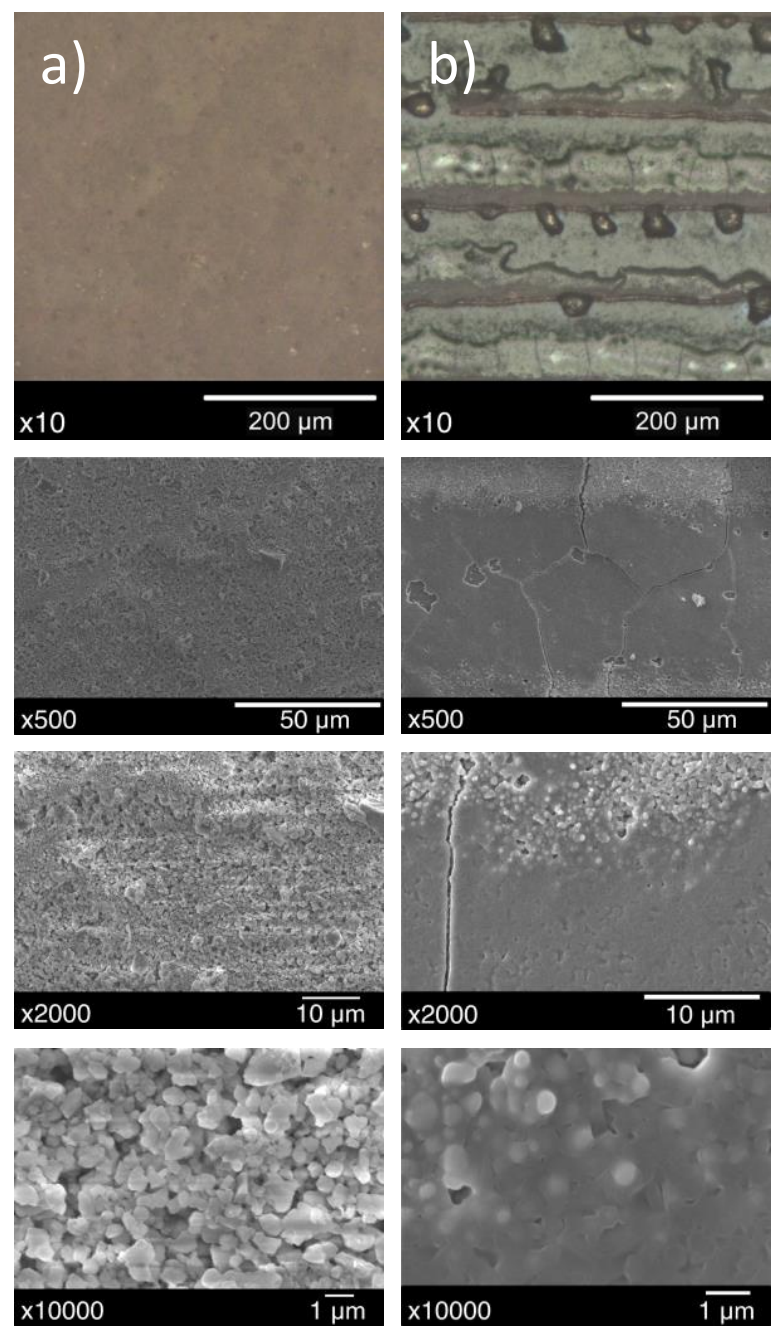

Figure 5. Optical microscopy (top view of the films) and SEM micrographs at various magnifications for a sintering cordon for two samples with two different copper quantities (left column: $x=0.1$; right column: $x=0.5$ ).

These experimental results can be related to the data of the numerical analyses displayed in Figure 6. Four values of absorptance $a$ (eq. (2)) were chosen $(0.05,0.08,0.1$, and 0.12); the laser power and the scanning speed were varied between $40 \mathrm{~W}$ and $90 \mathrm{~W}$ and 0.2 and $0.45 \mathrm{~m} . \mathrm{s}^{-}$ ${ }^{1}$, respectively. The maximum temperatures reached during the calculated irradiation process are mapped against the laser power and scanning speed in Figures 6a-d. The maximum temperature achieved (Figures 6e-f) was proportional to the absorptance. By comparing the maximal temperatures for a power/speed laser couple fixed at $60 \mathrm{~W} / 0.30 \mathrm{~m} . \mathrm{s}^{-1}$ (time profiles shown in Figures 6e), it was determined the maximum temperatures were strictly proportional 
to the absorptance (linear law) (Figure 6f), which gave the percentage of absorbed power for given applied laser power.
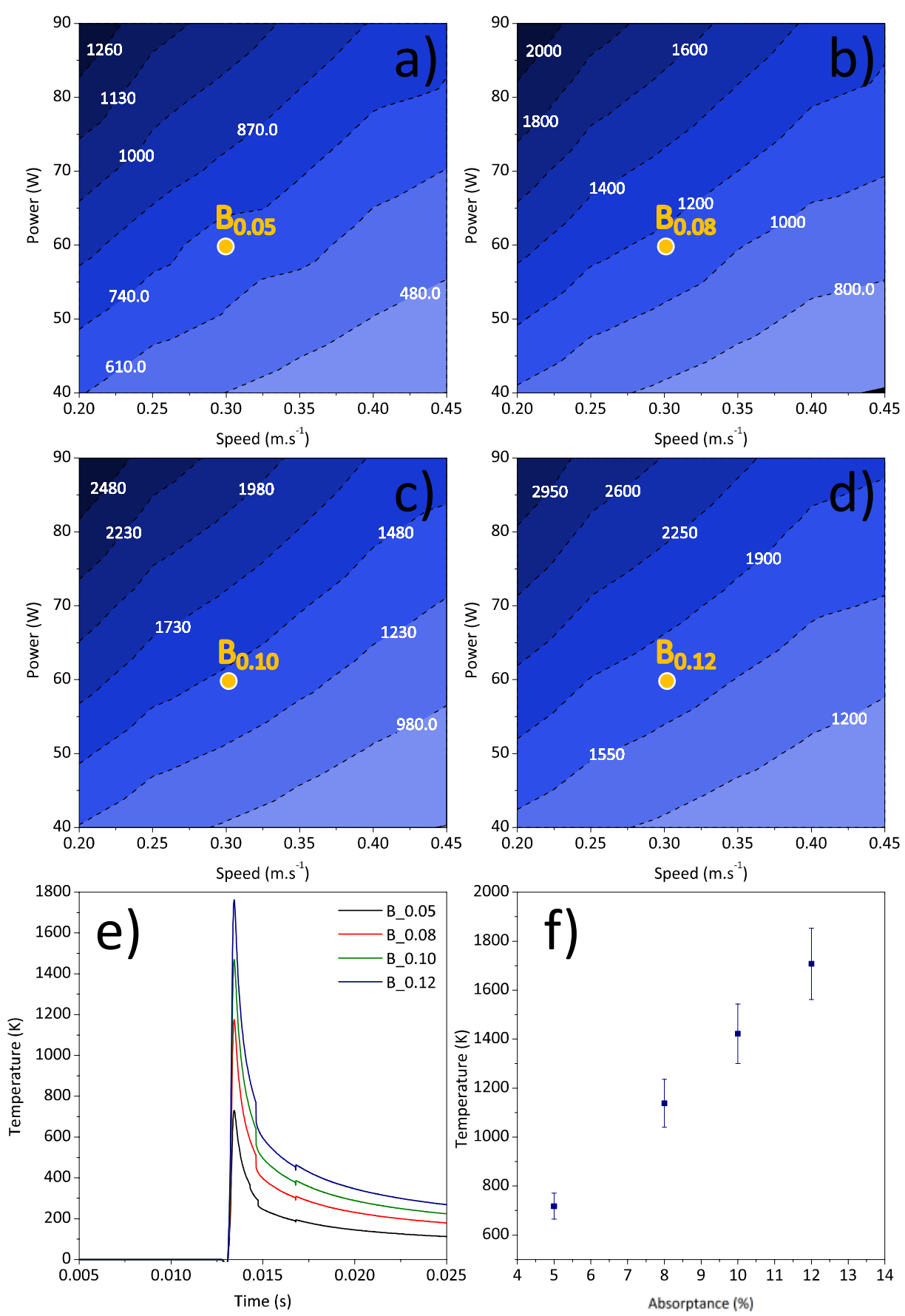

Figure 6.a-d) Maps of maximum temperature simulated for various Cu-doped HA-irradiated samples according to their absorptance (0.05, 0.08, 0.10, and 0.12); e) Time-temperature plots for the median point of the irradiated line with laser power and scan rate represented by the four central points in Figs. $a-d ; f)$ plot of the average maximum temperature and its standard deviation versus the sample absorptance obtained from maps in a-d. 
Experimental and numerical results indicate the major impact of laser power absorption by the material on the thermal elevation of the irradiated area and thus on the sintering quality. Therefore, the absorptance of the material is a key parameter for controlling the SLS process that has to be accurately determined for superior process control.

\subsection{Impact of film thickness}

For the $\mathrm{Cu}$-doped HA compound with $x=0.5$, the film thickness influence was studied from 2, 6, and 10 successive layers. Figure 7 establishes that the film thickness is proportional to the number of successive coating steps. Ten successive layers resulted in a $25 \mu \mathrm{m}$ thickness, as depicted by the SEM cross-section micrograph.

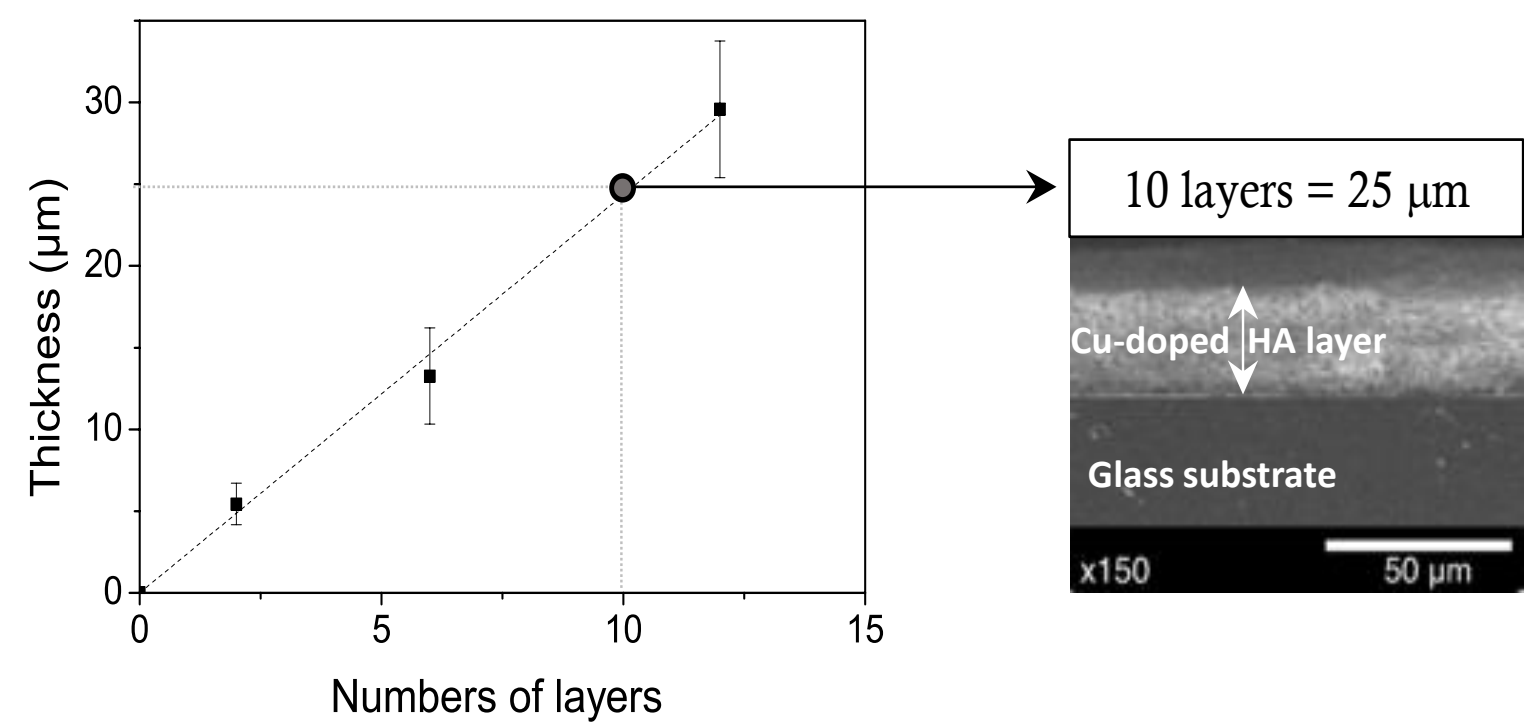

Figure 7. Evolution of the film thickness with the number of dip-coating steps (or layers). SEM pictures allow measuring the thickness of the film.

The optical and SEM micrographs of irradiated zones of two films with different thicknesses (2 and 5 layers) are reported in Figure 8. With a magnification allowing for the observation of three horizontal laser-treated lines, the optical micrographs clearly show the thicker film's irradiated areas. Moreover, the thicker film demonstrates a much better contrast between irradiated and non-irradiated zones than the thinner film, where this effect is less clear. For both films, the irradiated area colour was modified due to the thermal elevation (slighter in the thinner film) consecutive to irradiation. This colour change means that the maximum reached temperatures during laser irradiation were higher than the pre-annealing treatment temperature 
(operated at $1100^{\circ} \mathrm{C}$ ). The SEM micrographs confirm that the sintering neck formation between particles is well observed only on the thicker film, which also exhibits very dense areas, suggesting that the samples would have even partially melted during laser irradiation; much less effective sintering in the thinner film can be observed by the slightly densified powder bed.
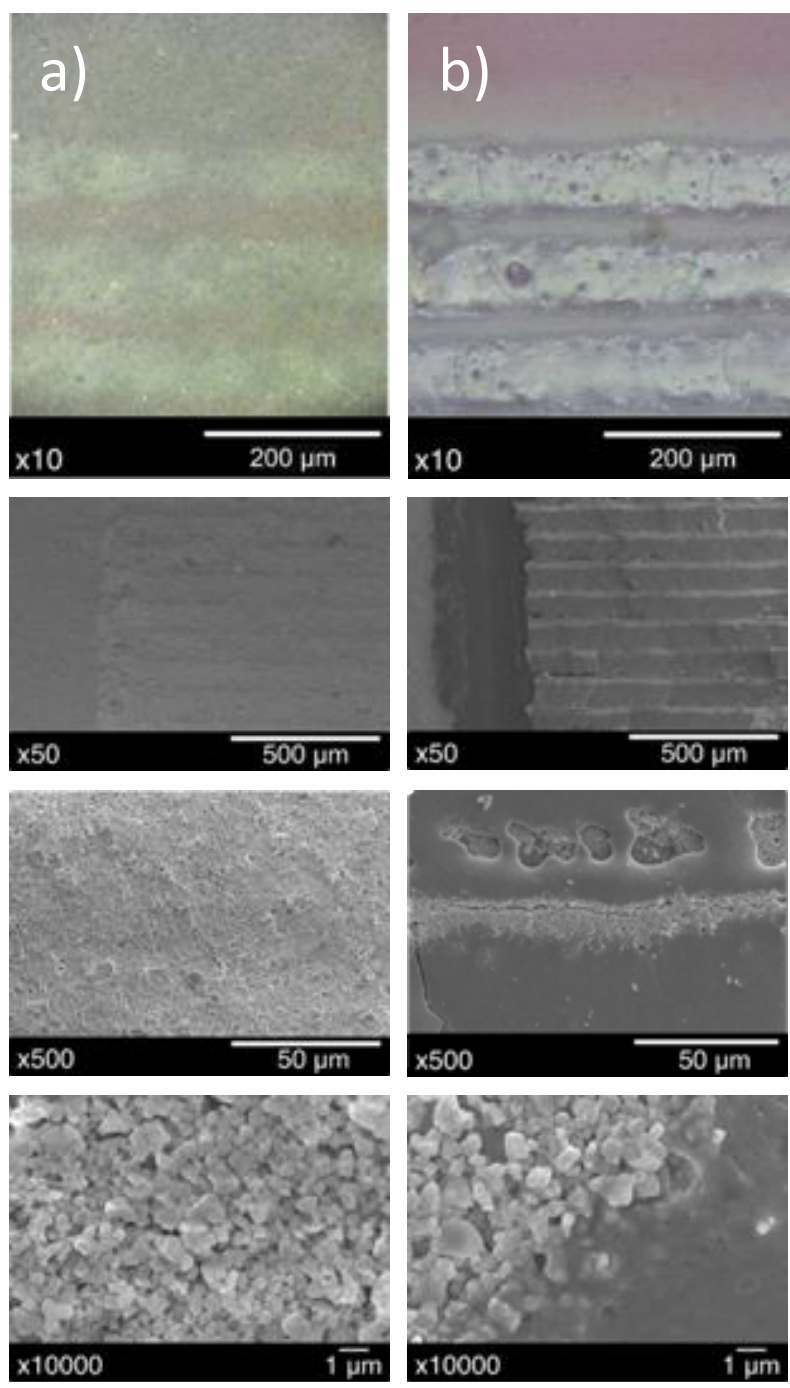

Figure 8. Optical microscopy and SEM pictures at various magnifications from a sintering cordon for two samples with different thicknesses (2 layers for left column and 5 layers for right column).

Numerical calculations were performed on films with various thicknesses ranging from 2 to $100 \mu \mathrm{m}$ and considering that the heated volume from laser irradiation can be cylindrical or semi-ellipsoid in shape with a fixed diameter of $25 \mu \mathrm{m}$ in both cases. Regardless of the heated volume shape, two regimes can be distinguished on the curves describing the variation in the maximum temperature reached by the sample versus the sample thickness (Figure 9). In the first regime, when the film thickness is lower than the heated volume depth, the temperatures 
reached during the laser/matter interaction are predicted to increase with the film thickness linearly. This tendency stops when the film thickness becomes larger than the heated volume depth, after which a plateau is observed of the maximum temperature resulting from laser irradiation. Thus, while the film thickness limits the heated volume, the glass substrate does not absorb at the laser wavelength so the thickness can control the maximum temperature of the $\mathrm{Cu}$-doped HA material during the SLS process. Hence, numerical simulation confirms the experimental characterisations. Additionally, Figure 9 suggests the effect is more drastic for the heated volume for semi-ellipsoid than cylindrical shapes. Furthermore, considering various heated volumes (for illustration, two heated cylindrical volumes with depths of $25 \mu \mathrm{m}$ and 60 $\mu \mathrm{m})$, we predicted that the smaller the heated volume, the higher the maximum temperature.

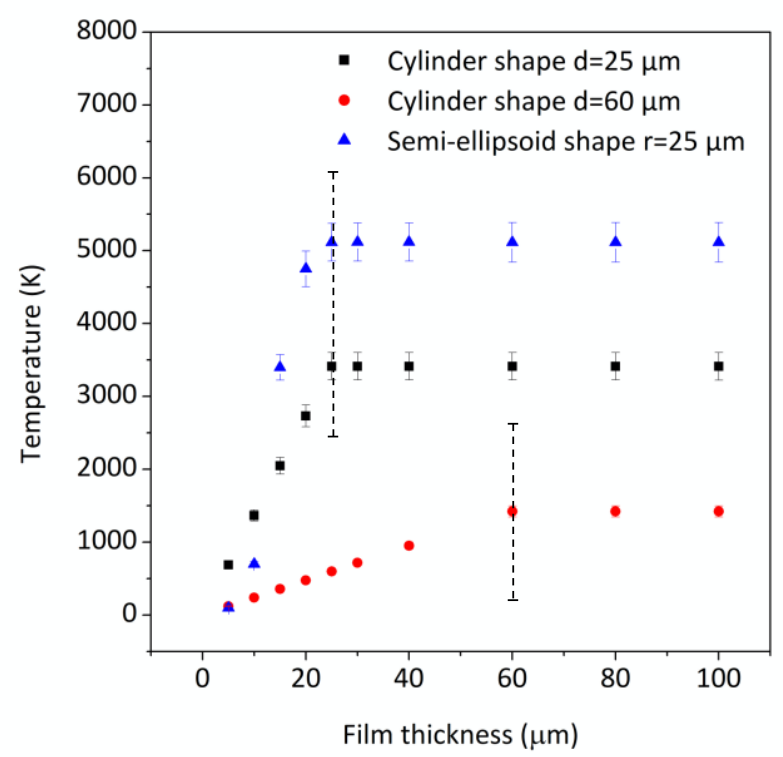

Figure 9. Evolution of the computed maximum temperature reached during laser irradiation by the sample films according to the shape and depth of the heated volume versus film thickness.

\subsection{Impact of laser scanning speed and laser power at constant total linear energy}

The impact of laser power and laser scanning speed was studied for linear iso-energetic ( $\left.\mathrm{J} . \mathrm{m}^{-1}\right)$ irradiation, for a constant power/speed ratio in our experimental investigations (SEM micrographs reported in Figure 10). 


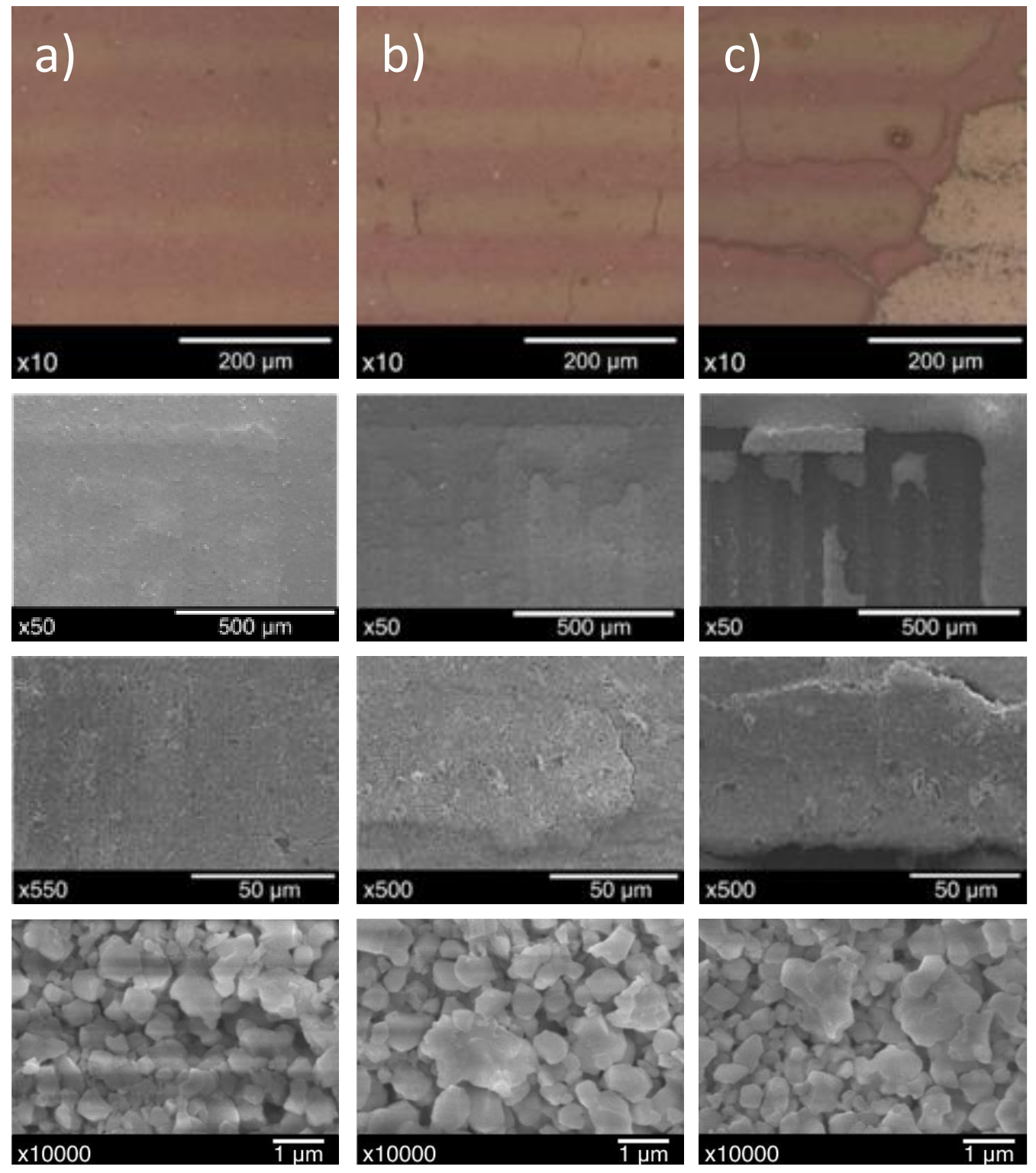

Figure 10. Optical micrographs and SEM micrographs at various magnifications taken on irradiated zones using iso-energetic laser excitation but with different power/scanning speed laser parameters: $0.20 \mathrm{~m} \cdot \mathrm{s}^{-1} / 40 \mathrm{~W}(\mathrm{a}) ; 0.21 \mathrm{~m} \cdot \mathrm{s}^{-1} / 42 \mathrm{~W}(\mathrm{~b})$ and $0.22 \mathrm{~m} \cdot \mathrm{s}^{-1} / 44 \mathrm{~W}(\mathrm{c})$.

Increasing the laser energy received by the sample produces a rise in the maximum temperatures, which is described in the literature [16,20,32]. Moreover, the temperature mappings (reported in Figure 11a) extracted from our numerical simulations for varying laser energies, laser scanning speeds ranging from $0.20 \mathrm{~m} . \mathrm{s}^{-1}$ up to $0.45 \mathrm{~m} . \mathrm{s}^{-1}$, and laser power from $40 \mathrm{~W}$ up to $90 \mathrm{~W}$ confirm these observations. The irradiated zone temperature gain of the film is estimated to be approximately $1000^{\circ} \mathrm{C}$ and $2000^{\circ} \mathrm{C}$ using a scanning speed of $0.30 \mathrm{~m} . \mathrm{s}^{-1}$ with laser powers of 40 and $90 \mathrm{~W}$, respectively; the film temperatures using a power equal to $60 \mathrm{~W}$ are predicted to be approximately $1800^{\circ} \mathrm{C}$ and $1000^{\circ} \mathrm{C}$ for scanning speeds of 0.20 and 0.45 
$\mathrm{m} . \mathrm{s}^{-1,}$ respectively. Consequently, maximum temperatures are roughly proportional to the energy received from laser irradiation (Figure 11b-c), consistent with a previous numerical study on the effect of absorptance.
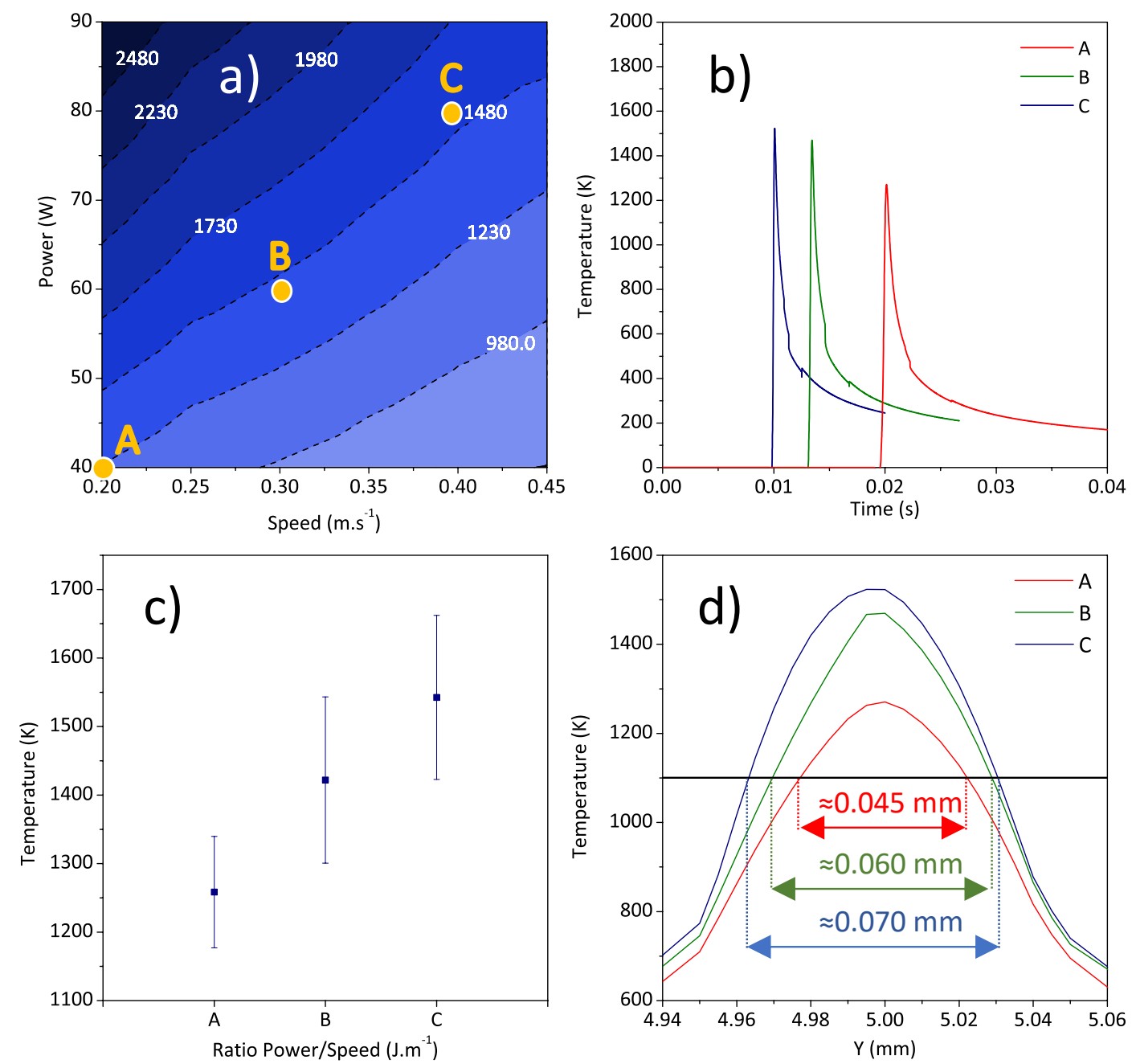

Figure 11. Maps of maximum temperature simulated according to laser power and laser scanning speed (a); evolution of temperature reached by the median point of the irradiated line for scanning speed and laser power corresponding to the $A, B$, and $C$ parameter sets (b); plot of the maximum temperature reached by the median point for parameter sets $A, B$, and $C(c)$; temperature profile recorded perpendicular to the lased line at the median point for parameter sets $A, B$, and $C(d)$.

We also examine whether the laser power and laser speed can have an impact while the laser power/scanning speed ratio is fixed to a single value. The optical micrographs of Figure 10 show an enhancement of the colour modification created by laser line irradiation with powder or scan rate. The colour change due to the sintering effect at a more elevated temperature than the pre-annealing treatment $\left(1100^{\circ} \mathrm{C}\right)$, confirmed by the corresponding SEM micrographs, is 
associated with an enlargement of the irradiated line width when the laser power and scanning speed are both increased. Furthermore, the emergence of cracks located at the interface of the irradiated and non-irradiated areas is visible for the medium and high parameter sets. The sintered lines separate from the substrate (delamination phenomenon) for the last set of parameters, i.e., highest speed and power parameter set.

Nonetheless, SEM images reveal $\mathrm{Cu}$-doped HA particles without the presence of sintering necks regardless of the laser irradiation parameter set. Even though the delamination or decohesion observed for high laser power and high scanning speed strongly suggests that partial densification of the irradiated zones has occurred, leading to at least mechanical cohesion of these irradiated lines. Although the sintering advancement degree seems quite similar (more rigorously, not so different) for the three-parameter sets that have been examined, the presence or absence of cracks depending on the parameter set shows that the mechanical stresses are not the same for the three samples. When high laser power is combined with high scanning speed, the enlargement of the lased line width reflects a more extensive spread of heat transfer due to the achievement of a higher maximum temperature in the centre of the irradiated zone. Crack presence reflects more important internal stresses for these last parameter sets than for the first two sets, which could be generated by faster shrinkage associated with the sintering phenomenon [35] or thermal expansion differences with the substrate.

These experimental outcomes were compared with numerical simulation findings for three isoenergetic irradiations (points A, B, and C in Figure 11a). The temperatures gained by the HA films increase with increasing laser power and laser scanning speed. Moreover, the increase in the maximum temperature at the centre of the exposed area is well associated with an enlargement of its width (as shown from our simulation reported in Figure 11d), precisely as observed experimentally (Figure 10). Therefore, it can be concluded that the increase in laser power and laser scanning velocity at constant linear energy causes an increase in the maximum temperature gained by the sample during the SLS process, with an increase in the heated volume and an increase in the mechanical stresses (tensile stresses at the interface between irradiated and non-irradiated areas). The increase in the thin film sample mechanical stresses can be related to excessively fast material shrinkage upon cooling after the sintering process compared to more elevated temperatures. A higher rate of volume shrinkage of the $\mathrm{Cu}$-doped $\mathrm{HA}$ films produced by material sintering does not allow the relaxation of stresses, and the film suffers crack formation or substrate/film delamination. 


\section{Conclusion}

Pure Cu-doped HA compounds obtained as powders after pre-annealing at $1100^{\circ} \mathrm{C}$, prepared as thin films with controlled thicknesses from dip coating on a glass substrate, were irradiated by an ytterbium laser (1.070 $\mu \mathrm{m}$ wavelength). Experimental laser irradiation was performed by modifying both the material (absorptivity from the copper concentration and film thickness) and laser source (scanning velocity and power) parameters. These experimental results were systematically compared to numerical modelling to predict the maximum temperature produced on the film by laser irradiation. First, it was revealed that the thermal conductivity and the radiative heat loss drive the temperature history since convective heat losses have negligible effects. The maximum temperatures reached upon laser irradiation are tuneable by material parameters such as the film thickness or the absorptance. The temperatures are directly proportional to the absorptance, whereas they can be limited by decreasing the film thickness below the heated volume depth. Finally, for constant linear energy, raising the laser power and scan rate (i.e., using different laser speeds and laser power parameters but with the same power/speed ratios), crack formation or film delamination from the substrate can be initiated. Numerical simulation supports that cracks can appear because higher temperatures and gradients are reached when high power combined with high scanning velocity is used. This last observation opens the possibility of avoiding one of the main drawbacks of the SLS process: crack formation. 


\section{References}

[1] N. Eliaz, N. Metoki, Calcium Phosphate Bioceramics: A Review of Their History, Structure, Properties, Coating Technologies and Biomedical Applications, Materials. 10 (2017) 334. https://doi.org/10.3390/ma10040334.

[2] S.V. Dorozhkin, Bioceramics of calcium orthophosphates, Biomaterials. 31 (2010) 14651485. https://doi.org/10.1016/j.biomaterials.2009.11.050.

[3] M. Šupová, Substituted hydroxyapatites for biomedical applications: A review, Ceram. Int. 41 (2015) 9203-9231. https://doi.org/10.1016/j.ceramint.2015.03.316.

[4] J.T.B. Ratnayake, M. Mucalo, G.J. Dias, Substituted hydroxyapatites for bone regeneration: A review of current trends, J. Biomed. Mater. Res. B Appl. Biomater. 105 (2017) 1285-1299. https://doi.org/10.1002/jbm.b.33651.

[5] M. Lasgorceix, E. Champion, T. Chartier, Shaping by microstereolithography and sintering of macro-micro-porous silicon substituted hydroxyapatite, J. Eur. Ceram. Soc. 36 (2016) 1091-1101. https://doi.org/10.1016/j.jeurceramsoc.2015.11.020.

[6] S. Bose, Understanding of dopant-induced osteogenesis and angiogenesis in calcium phosphate ceramics, 31 (2013) 12. https://doi.org/10.1016/j.tibtech.2013.06.005.

[7] R.B. Unabia, S. Bonebeau, R.T. Candidato, L. Pawłowski, Preliminary study on copperdoped hydroxyapatite coatings obtained using solution precursor plasma spray process, Surf. Coat. Technol. 353 (2018) 370-377. https://doi.org/10.1016/j.surfcoat.2018.09.008.

[8] Y. Huang, X. Zhang, R. Zhao, H. Mao, Y. Yan, X. Pang, Antibacterial efficacy, corrosion resistance, and cytotoxicity studies of copper-substituted carbonated hydroxyapatite coating on titanium substrate, J. Mater. Sci. 50 (2015) 1688-1700. https://doi.org/10.1007/s10853-014-8730-1.

[9] P. Rider, Ž.P. Kačarević, S. Alkildani, S. Retnasingh, R. Schnettler, M. Barbeck, Additive Manufacturing for Guided Bone Regeneration: A Perspective for Alveolar Ridge Augmentation, Int. J. Mol. Sci. 19 (2018). https://doi.org/10.3390/ijms19113308.

[10] L. Ferrage, G. Bertrand, P. Lenormand, D. Grossin, B. Ben-Nissan, A review of the additive manufacturing (3DP) of bioceramics: alumina, zirconia (PSZ) and hydroxyapatite, J. Aust. Ceram. Soc. 53 (2017) 11-20. https://doi.org/10.1007/s41779016-0003-9.

[11] N. Travitzky, A. Bonet, B. Dermeik, T. Fey, I. Filbert-Demut, L. Schlier, T. Schlordt, P. Greil, Additive Manufacturing of Ceramic-Based Materials, Adv. Eng. Mater. 16 (2014) 729-754. https://doi.org/10.1002/adem.201400097.

[12] I. Gibson, D. Rosen, B. Stucker, Additive Manufacturing Technologies, Springer New York, New York, NY, 2015. https://doi.org/10.1007/978-1-4939-2113-3.

[13] R.L. Coble, Sintering Crystalline Solids. I. Intermediate and Final State Diffusion Models, J. Appl. Phys. 32 (1961) 787-792. https://doi.org/10.1063/1.1736107.

[14] X. Liu, M. Boutaous, S. Xin, Numerical modeling and simulation of selective laser sintering in polymer powder bed, Université de Lyon, 2017. https://tel.archivesouvertes.fr/tel-01920677.

[15] R.K. Bordia, S.-J.L. Kang, E.A. Olevsky, Current understanding and future research directions at the onset of the next century of sintering science and technology, J. Am. Ceram. Soc. 100 (2017) 2314-2352. https://doi.org/10.1111/jace.14919.

[16] A. Hussein, L. Hao, C. Yan, R. Everson, Finite element simulation of the temperature and stress fields in single layers built without-support in selective laser melting, Mater. Des. 1980-2015. 52 (2013) 638-647. https://doi.org/10.1016/j.matdes.2013.05.070. 
[17] S. Kumar, Selective laser sintering: A qualitative and objective approach, JOM. 55 (2003) 43-47. https://doi.org/10.1007/s11837-003-0175-y.

[18] C. Gao, B. Yang, H. Hu, J. Liu, C. Shuai, S. Peng, Enhanced sintering ability of biphasic calcium phosphate by polymers used for bone scaffold fabrication, Mater. Sci. Eng. C. 33 (2013) 3802-3810. https://doi.org/10.1016/j.msec.2013.05.017.

[19] L. Ferrage, G. Bertrand, P. Lenormand, Dense yttria-stabilized zirconia obtained by direct selective laser sintering, Addit. Manuf. $21 \quad$ (2018) 472-478. https://doi.org/10.1016/j.addma.2018.02.005.

[20] I. Yadroitsev, Ph. Bertrand, I. Smurov, Parametric analysis of the selective laser melting

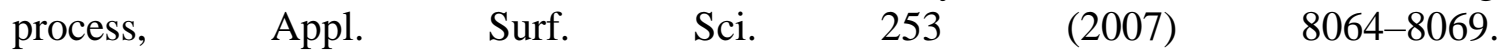
https://doi.org/10.1016/j.apsusc.2007.02.088.

[21] P. Peyre, P. Aubry, R. Fabbro, R. Neveu, A. Longuet, Analytical and numerical modelling of the direct metal deposition laser process, J. Phys. Appl. Phys. 41 (2008) 025403. https://doi.org/10.1088/0022-3727/41/2/025403.

[22] P. Conti, F. Cianetti, P. Pilerci, Parametric Finite Elements Model of SLM Additive Manufacturing process, Procedia Struct. Integr. 8 (2018) 410-421. https://doi.org/10.1016/j.prostr.2017.12.041.

[23] L. Dong, A. Makradi, S. Ahzi, Y. Remond, Three-dimensional transient finite element analysis of the selective laser sintering process, J. Mater. Process. Technol. 209 (2009) 700-706. https://doi.org/10.1016/j.jmatprotec.2008.02.040.

[24] N.K. Tolochko, M.K. Arshinov, A.V. Gusarov, V.I. Titov, T. Laoui, L. Froyen, Mechanisms of selective laser sintering and heat transfer in Ti powder, Rapid Prototyp. J. 9 (2003) 314-326. https://doi.org/10.1108/13552540310502211.

[25] A.V. Gusarov, I. Smurov, Radiation transfer in metallic powder beds used in laser processing, J. Quant. Spectrosc. Radiat. Transf. 111 (2010) 2517-2527. https://doi.org/10.1016/j.jqsrt.2010.07.009.

[26] N.K. Tolochko, Y.V. Khlopkov, S.E. Mozzharov, M.B. Ignatiev, T. Laoui, V.I. Titov, Absorptance of powder materials suitable for laser sintering, Rapid Prototyp. J. 6 (2000) 155-161. https://doi.org/10.1108/13552540010337029.

[27] Y. Li, D. Gu, Parametric analysis of thermal behavior during selective laser melting additive manufacturing of aluminum alloy powder, Mater. Des. 63 (2014) 856-867. https://doi.org/10.1016/j.matdes.2014.07.006.

[28] P. Peyre, Y. Rouchausse, D. Defauchy, G. Régnier, Experimental and numerical analysis of the selective laser sintering (SLS) of PA12 and PEKK semi-crystalline polymers, J. Mater. $\quad$ Process. $\quad 225$ Technol. (2015) 326-336. https://doi.org/10.1016/j.jmatprotec.2015.04.030.

[29] A. Mokrane, M. Boutaous, S. Xin, Process of selective laser sintering of polymer powders: Modeling, simulation, and validation, Comptes Rendus Mécanique. 346 (2018) 10871103. https://doi.org/10.1016/j.crme.2018.08.002.

[30] L. Xin, M. Boutaous, S. Xin, D.A. Siginer, Numerical modeling of the heating phase of the selective laser sintering process, Int. J. Therm. Sci. 120 (2017) 50-62. https://doi.org/10.1016/j.ijthermalsci.2017.05.017.

[31] L. Xin, M. Boutaous, S. Xin, D.A. Siginer, Multiphysical modeling of the heating phase in the polymer powder bed fusion process, Addit. Manuf. 18 (2017) 121-135. https://doi.org/10.1016/j.addma.2017.10.006.

[32] D. Riedlbauer, M. Drexler, D. Drummer, P. Steinmann, J. Mergheim, Modelling, simulation and experimental validation of heat transfer in selective laser melting of the polymeric material PA12, Comput. Mater. Sci. 93 (2014) 239-248. https://doi.org/10.1016/j.commatsci.2014.06.046. 
[33] S. Raynaud, Synthèse, frittage et propriétés mécaniques de phosphates de calcium dans le système hydroxyapatite - phosphate tricalcique, These de doctorat, Limoges, 1999. http://www.theses.fr/1999LIMO0035 (accessed November 23, 2020).

[34] L. Ma, H. Bin, Temperature and stress analysis and simulation in fractal scanning-based laser sintering, Int. J. Adv. Manuf. Technol. 34 (2007) 898-903. https://doi.org/10.1007/s00170-006-0665-5.

[35] J.R. Carazzone, C.L. Martin, Z.C. Cordero, Crack initiation, propagation, and arrest in sintering powder aggregates, J. Am. Ceram. Soc. 103 (2020) 4754-4773. https://doi.org/10.1111/jace.17170. 


\section{Appendix}

\section{A1. Impact of convective/radiative heat losses}

In Figure A1, the maximum temperature is plotted versus time for the median point of an irradiation line of $1 \mathrm{~cm}$ long, using three different simulation hypotheses for the heat losses (all other parameters kept fixed): (i) both radiative losses and convective losses at both interfaces between air and film and film and substrate and radiative; (ii) only the convection loss at the film/air interface plus the radiative loss and (iii) only radiative loss.

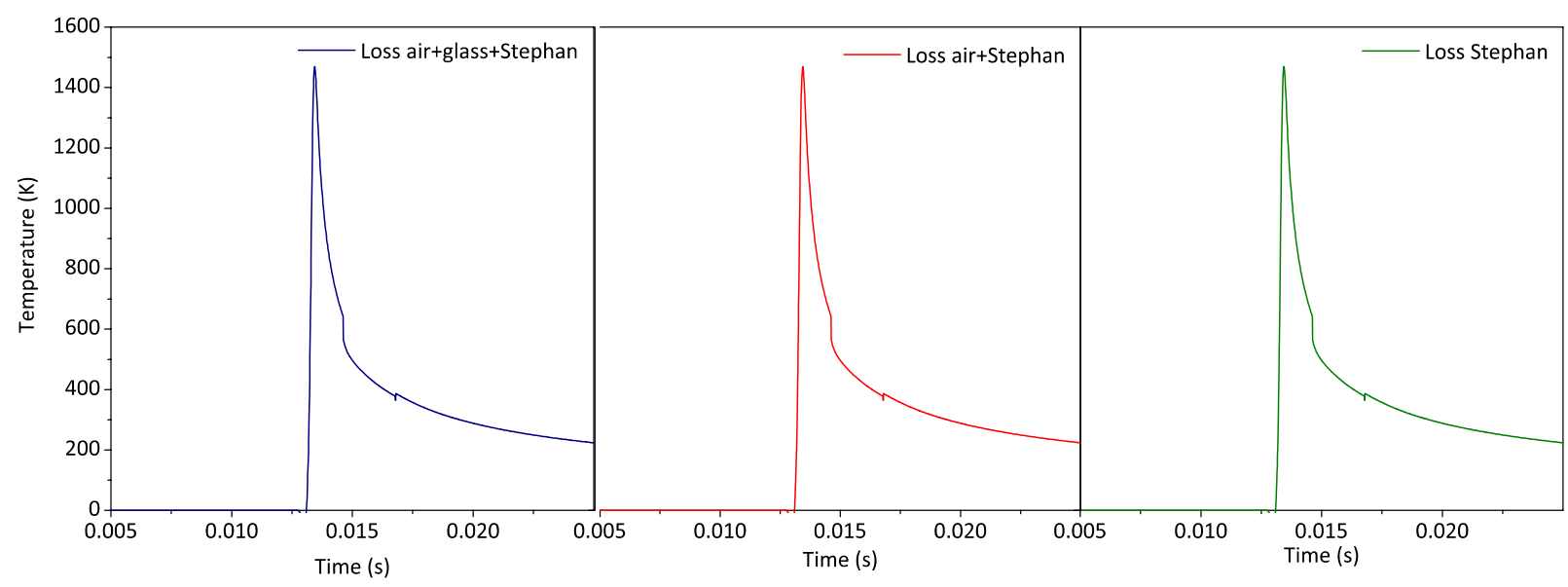

Figure A1. Evolution of temperature reached by the median point of the irradiated line considering different heat loss mechanisms.

It can be seen that the maximum temperatures reached (about $1400^{\circ} \mathrm{C}$ ) when the laser passes over the median point are identical regardless convective heat losses are included or not in the computation. The plots, from the asymmetric temperature profile of this median point shows that the elevation of temperature due to conduction before the laser passes over the analyzed point is negligible; on the other hand, the temperature decrease after the laser spot as passed the median point is rapid from $1400^{\circ} \mathrm{C}$ to $500^{\circ} \mathrm{C}$ and then much slower. Anyway, this cooling rate does not seem to be piloted by convective heat losses. It can be concluded that due to the high speed of the sintering process (with a very short duration of the thermal elevation, about $2 \mathrm{~ms}$ beyond $500^{\circ} \mathrm{C}$ ), the convective heat losses at both air/film and film/substrate interfaces are negligible. They will therefore be neglected in all other numerical analysis presented here. 